\title{
Spatially Selective Assembly of Quantum Dot Light Emitters in an LED Using Engineered Peptides
}

\author{
Hilmi Volkan Demir, ${ }^{\dagger, \perp, *}$ Urartu Ozgur Safak Seker, ${ }^{\dagger, \perp}$ Gulis Zengin, $^{\dagger}$ Evren Mutlugun, $^{\dagger}$ Emre Sari, $^{\dagger}$ \\ Candan Tamerler, ${ }^{\ddagger, \S}$ and Mehmet Sarikaya ${ }^{\S, *}$
}

\begin{abstract}
${ }^{\dagger}$ Department of Electrical and Electronics Engineering, Department of Physics and UNAM, Institute of Materials Science and Nanotechnology, Bilkent University, 06800 Ankara, Turkey, ${ }^{\ddagger}$ Molecular Biology and Genetics, MOBGAM, Istanbul Technical University, Maslak 34469, Istanbul, Turkey, ${ }^{\S}$ Genetically Engineered Materials Science and Engineering Center, Department of Materials Science and Engineering, University of Washington, Seattle, Washington 98105, United States, and ${ }^{\perp}$ Luminous! Center of Excellence for Semiconductor Lighting and Displays, School of Electrical and Electronic Engineering, Microelectronics Division, School of Physical and Mathematical Sciences, Physics and Applied Physics Division, Nanyang Technological University, 639798 Singapore
\end{abstract}

S emiconductor quantum dots (QDots), such as colloidal nanocrystals made of $\mathrm{CdSe} / \mathrm{ZnS}$ in core-shell architecture, have been extensively utilized as functional materials in a variety of photonic and electronic device platforms. ${ }^{1-5}$ QDots are useful because of their optical and electronic properties including size-tunable optical emission, high quantum yield, and photostability. Recently, for example, capacity of QDots has also been demonstrated for white light generation and tunability in color-conversion white-light-emitting diodes (LEDs). ${ }^{6-10}$ To fully exploit useful characteristics of QDots, however, two significant limitations still have to be overcome simultaneously during the processing of real-life device settings. First, QDots have to be assembled with specific spatial distribution in space with respect to one another for enhanced functionality; and second, the dots have to be positioned at desired locations, at the micrometer scale, on devices that typically have several different material components.

In previous studies, the distribution problem is partially solved by performing the assembly of QDots, in host polymers (e.g., PMMA), ${ }^{11,12}$ layer-by-layer assembly using thin films of polyelectrolytes (e.g., PAA and PSS), ${ }^{13-15}$ or the particles were assembled as closely packed self-aggregates without using a host scaffold or an additional film. None of these approaches, however, has the potential of assembling the QDots in prespecified locations on a practical device, which usually has multilateral micropatterned architectures. For instance, a photonic LED platform consists of at least three materials, including metal (e.g., gold),
ABSTRACT Semiconductor nanocrystal quantum dots are utilized in numerous applications in nano- and biotechnology. In device applications, where several different material components are involved, quantum dots typically need to be assembled at explicit locations for enhanced functionality. Conventional approaches cannot meet these requirements where assembly of nanocrystals is usually material-nonspecific, thereby limiting the control of their spatial distribution. Here we demonstrate directed self-assembly of quantum dot emitters at material-specific locations in a color-conversion LED containing several material components including a metal, a dielectric, and a semiconductor. We achieve a spatially selective immobilization of quantum dot emitters by using the unique material selectivity characteristics provided by the engineered solid-binding peptides as smart linkers. Peptide-decorated quantum dots exhibited several orders of magnitude higher photoluminescence compared to the control groups, thus, potentially opening up novel ways to advance these photonic platforms in applications ranging from chemical to biodetection.

KEYWORDS: directed assembly · self-assembly - material selectivity · inorganic binding peptides $\cdot$ quantum dots $\cdot$ LEDs $\cdot$ optoelectronics

semiconductor (e.g., InAlGaN), and a dielectric (e.g., silica) in a complex patterned architecture. $^{6,16}$ In a standard LED, by driving a forward current through its metal contacts, electrons and holes are injected into the semiconductor. The recombination of electrons and holes in the active region gives rise to emission of light by spontaneous process in every direction. The resulting photons are coupled out through an optical emission cone determined by the ratio of the refractive indices of the semiconductor with respect to air and the location of charge injection into the active region. The working principle of color-conversion LEDs involves optically pumping of their integrated luminophors, such as phosphors and QDots, through emission from an optical window. The subsequent collection of the photoluminescence of luminophors and electroluminescence of LED dictate the

\begin{abstract}
* Address correspondence to volkan@bilkent.edu.tr, hvdemir@ntu.edu.sg, sarikaya@u.washington.edu.
\end{abstract}

Received for review November 18, 2010 and accepted February 23, 2011.

Published online February 23, 2011 $10.1021 / \mathrm{nn} 103127 \mathrm{v}$

C 2011 American Chemical Society 
operation of the device. Immobilization of QDots only on the targeted optical windows on a LED chip is, therefore, essential simply because only the QDots selfassembled at the desired locations can be efficiently pumped. The QDots assembled on the metal or the semiconductor regions out of the emission cone are not pumped and, therefore, do not contribute to the overall luminescence of the hybrid device. As a consequence, in addition to avoiding the excess use of QDots unnecessarily accumulated in undesired areas of the device, another, more important, problem is also eliminated. In fact, the particles, assembled on metal contacts, could hinder the connection between contacts and wire or probe to the electric supply, thereby making it difficult to drive the LED if their metal connections are made after the immobilization of QDots. Eliminating such a problem in real devices, at large dimensional scales, is impractical during both fabrication and performance, requiring additional steps including the use of the traditional patterning techniques involving multiple lithography steps and cleaning procedures, or always necessitating the wiring of the devices beforehand. ${ }^{17-19}$ To overcome these limitations, our unique approach utilizes inorganic solid-binding peptides that are molecular linkers, functionalizing the QDots, and have specific material selectivity property, essential for selective spatial assembly. To demonstrate how this approach works, we show here the directed assembly of peptide-functionalized QDots only on the targeted optical windows of a nanocrystal-based color-conversion LED. Since the fabrication approach is straightforward via a simple patterning, potentially, it could be easily adapted to other, and more sophisticated, chip configurations such as modulators, ${ }^{20}$ photovoltaics, ${ }^{21,22}$ photodetectors, ${ }^{23}$ and optical memories. ${ }^{24}$

The genetically engineered peptides for inorganics (GEPI) have been biocombinatorially selected using phage or cell surface display approaches..$^{25-28}$ These short amino-acid-containing sequences are then characterized for their binding affinity and selectivity toward their robust utility. The solid-binding peptides are increasingly becoming ubiquitous molecular elements frequently utilized in the synthesis of inorganic materials and as linkers and assemblers of nanoscale objects. ${ }^{29-32}$ It is now also possible to in silico design a new generation of peptides with enhanced binding and material selectivity characteristics. ${ }^{33}$ In this work, we use such a designed peptide, the silica-binding peptide (QBP1) as silica-specific linkers for QDots. The QBP1 has a 12 amino acid sequence, PPPWLPYMPPWS, displaying binding affinity and free energy of binding values of $7.0 \times 10^{5} \mathrm{M}^{-1}$ and $8.0 \mathrm{kcal} / \mathrm{mol}$, respectively (see Supporting Information). When used alone, QBP1 is found to have a surface coverage of about $80 \%$ under equilibrium conditions. We used biotinylated peptide (i.e., QBP1-bio) as a molecular linker for streptavidin- functionalized QDot (i.e., SA-QDot) immobilization on the targeted regions on the device. This way, the peptide now features bifunctionality: silica binding coupled with molecular recognition of QDot through SA. In this study, we first explored the selectivity and specificity of the peptide-decorated QDot hybrids on silica, GaN, and gold surface to ensure their suitability for our optoelectronic device application. The specificity and affinity of these nanohybrids were found satisfying. Further we employed the nanohybrid structures of QDots to furnish LED devices with high selectivity. For the first time, we demonstrated the utilization of peptide-QDot nanohybrids on a real chip application. The results promise significant interest for opening new avenues in designing and building up novel nanostructure-based devices.

\section{RESULTS AND DISCUSSION}

We first explored the process of spatially selective assembly of QDots with high precision and surface coverage. We used two approaches to build a layer of QDots on the silica regions of the device (Figure 1). First, the peptides were assembled by incubating them on a polished $7 \mathrm{~mm} \times 7 \mathrm{~mm}$ silica substrate (with a rms roughness of $<2 \mathrm{~nm}$ ) overnight in an incubation chamber. The procedure involved a thorough washing step that removed nonspecifically bound and unbound peptides from the surface. At this stage, the QBP1-bio-decorated surface was ready for the assembly of SA-QDots, which was carried out by the drop-casting technique. Again, the sample was washed to remove any nonspecifically bound QDots to ensure stable and homogeneous QDot film on the surface. A negative control sample was also prepared by assembling the SA-QDot layer on the silica substrate directly in the absence of QBP1-bio (Figure 1a). The photoluminescence (PL) measurements of the samples, prepared with and without the QBP1-bio as the linker, reveal that they are not significantly different from each other. Furthermore, the optical behavior of these samples, which were prepared by the sequential assembly approach, was not reproducible. As we show in Figure $1 b$, the contrast variation in the fluorescence image exhibits a nonhomogeneous spreading of the SA-QDot on silica, with some of the regions on the chip exhibiting as low a PL signal as in the control group (Figure 1d). The problems revealed by these preliminary observations are related to the limited mass transfer of the QDots in solution. It, therefore, takes a substantial amount of incubation time for QDot-SA to diffuse and reach the QBP1-biofunctionalized silica surface regardless of the number of the available molecules on the target surface. The sequential way for targeted assembly of QDots on a silica substrate is a mass transfer-limited (or diffusionlimited) process as there is no further mixing involved to accelerate the mobility of the QDots reaching and, 


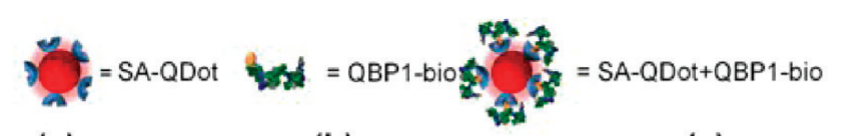

(a)

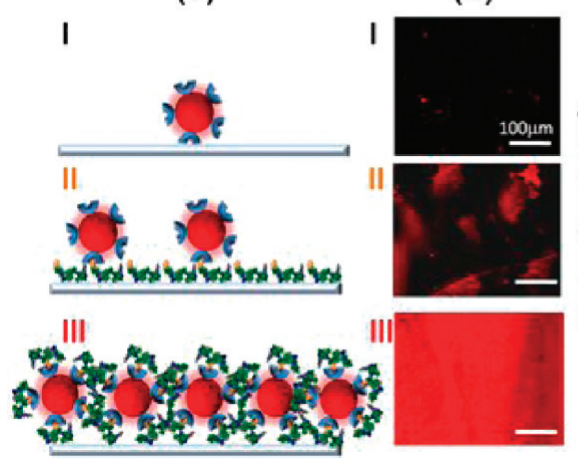

(c)

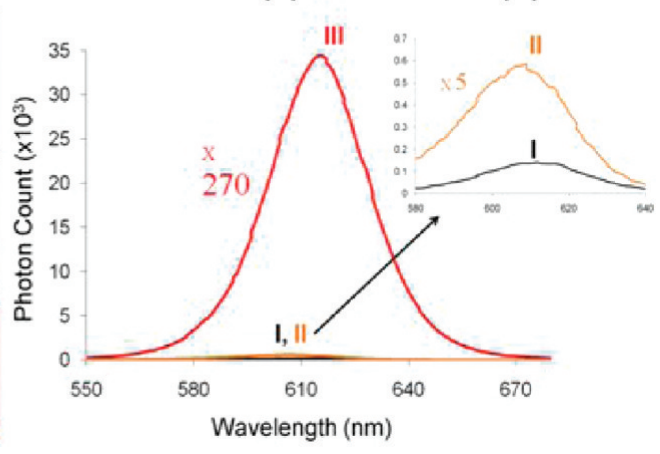

(d)

Figure 1. (a) Schematics of the assembly of QDots on silica: (i) without the use of silica (negative control), (ii) with QBP-bio bound to the silica surface, and (iii) with the QBP-bio bound to the (SA-QDot). The panels in (b) and (c) correspond to fluorescence images (at $610 \mathrm{~nm}$ ) and PL spectra, respectively. The image in b (i) reveals minimum coverage and c (i) very low CL signal. The approach in (ii), i.e., directing SA-QDot to QBP-bio-covered silica surface, has partial success, revealing nonhomogeneous, patchy coverage, shown in b (ii) and fairly low CL signal in c (ii). Finally, colloidal mixture of QBP-bio and SA-QDot leads to homogeneous coverage of the silica surface with continuous fluorescence in c (iii) and correspondingly high CL signal in C (iii).

then, binding to the surface through SA-bio molecular recognition.

To overcome the mass transfer problem described above, in the second approach, the QBP1-bio solution was first mixed with the QDot colloidal solution before the drop-casting step. This would allow QDot colloids and QBP1-bio molecules to be in close proximity in the buffer solution before the resultant homogeneous mixture is brought into contact with the silica substrate (see Supporting Information for detailed procedures). The samples prepared via the peptide-assisted assembly of the QBP1-QDot nanohybrids on the silica substrate resulted in a significant enhancement in the PL intensity compared to the control experiments (Figure 1d). Furthermore, particle assembly was highly uniform across hundreds of micrometer-wide regions on the sample surface (Figure 1c). This hybrid approach, as a consequence of the close control of the colloid mixture as well as the directed assembly of the QDots, resulted in a 2 orders of magnitude increase in $\mathrm{PL}$ intensity compared to the negative control and more than 1 order of magnitude more intensity over the sequential approach described above (Figure 1d).

In Figure 1, there is an obvious red shift in the peak emission wavelength of QDots decorated with QBP1 compared to the original peak emission wavelength of the QDots. This red shift is common when the dielectric constant of the environment around the QDots increases due to the conjugation of molecules. ${ }^{34,35}$ There is also a broadening in the emission spectrum of nonspecifically adsorbed streptavidin-coated quantum dots on the silica surface. This is attributed to the change of the hydrodynamic radius, which may be triggered by denaturation of streptavidin upon nonspecific binding on silica surface. This process may yield random change in the hydrodynamic radius of each QDot, causing a collectively broadened emission over a slightly wider range of wavelength.

In the next step, we demonstrate the binding specificity of QBP1-bio on an actual optoelectronic microchip surface, which was fabricated for solid-state-based lighting applications. ${ }^{5}$ On such an optoelectronic chip, there are at least three different types of patterned materials: dielectric (silica), metal (gold), and semiconductor (GaN) (Figure 2). The multimaterial characteristics of the chip, therefore, necessitate materialselective binding during the QDot assembly process. To target quantum dot assembly on specific regions of the patterned dielectric surface (silica regions), one must direct the QDots spatially and selectively onto the targeted regions, while avoiding both the metal and the semiconductor regions entirely. To ensure material specificity of the solid-binding peptide used in this work, we first characterized the binding specificity of the QBP1-bio construct alone on the silica-patterned gold substrate as well as gold-patterned silica substrate, where silica was used as the dielectric and gold as the conductor (Figure 2a). Preparation of the two different architectures allowed us to investigate the feasibility of decorating small features of patterned device mesas in complementary geometry in typical optoelectronic chips. Using the successful hybrid approach developed above, we incubated the samples with each geometry using the hybrid (QBP1-bio)/(SAQDot) colloid solution, as described. Next, the PL intensities of the samples from the metal and dielectric regions in both geometries were recorded as a function of wavelength (Figure $2 b$ ). Since the QBP1-bio is only selective to silica, but not to gold, the PL intensity peak from the silica regions of the chip is 9 times 

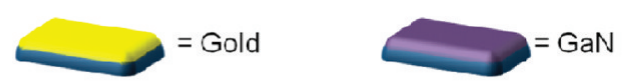

$=\mathrm{Si}_{x} \mathrm{O}_{y}$

(a)

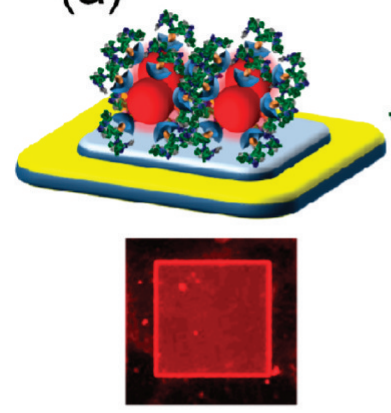

(b)

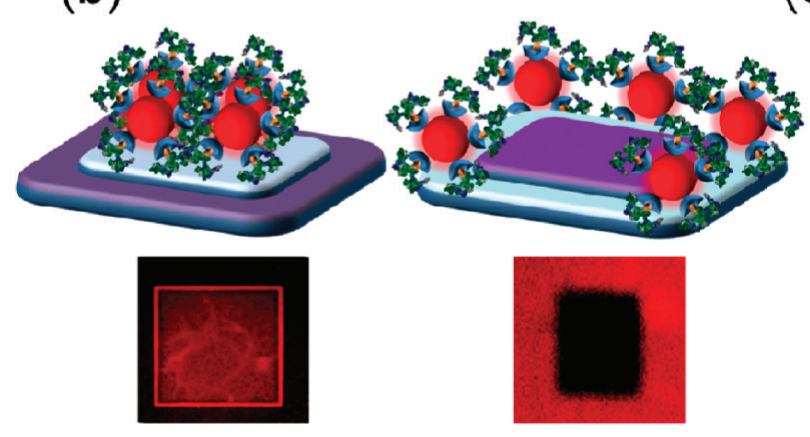

(c)
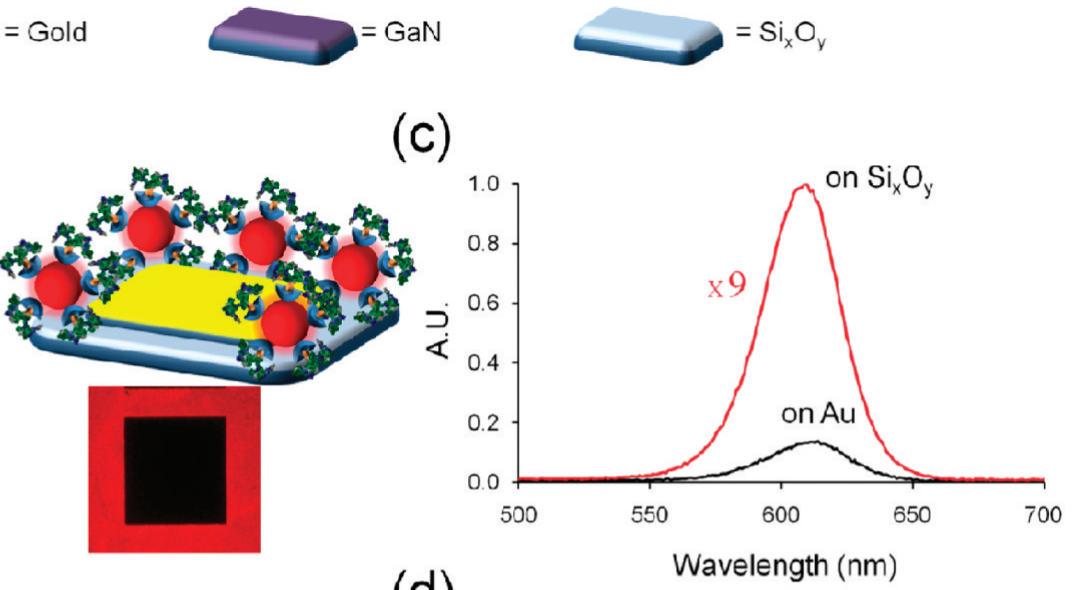

(d)

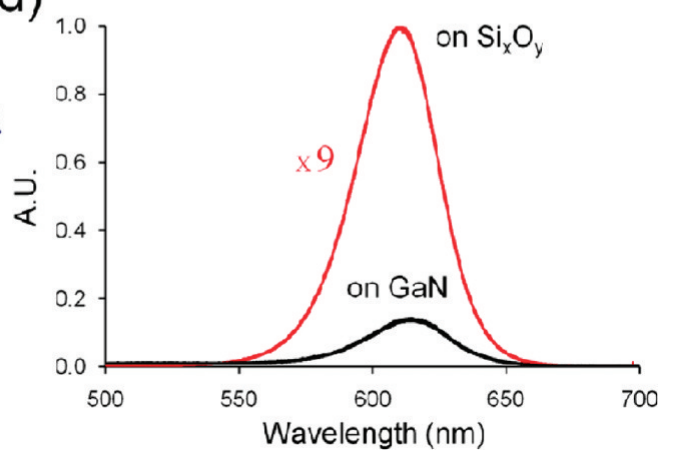

Figure 2. Schematics of QDot assembly on the silica region in a multimaterial containing mesa architecture (either ring or island), containing either (a) $\mathrm{Au} / \mathrm{SiO}_{x}$ or (b) $\mathrm{GaN} / \mathrm{SiO}_{x}$ using the (QBP1-bio)/(SA-QDot) hybrid molecular construct. The corresponding fluorescence images of peptide-assembled QDots are shown below the sketches. The reversal of color to red indicates that the dots assemble only on the silica regions in all cases. The photoluminescence spectra in (c) and (d) correspond to (a) and (b), revealing a 9-fold stronger signal (red) from the silica regions in all cases, compared to those from either gold or GaAs regions, respectively.

stronger than that from the gold regions. Similar to the gold/silica material selectivity tests above, we also carried out experiments to test the material selectivity of QBP1 between silica and GaN (Figure 2c), the latter being the critical semiconductor component of LED chips. As shown in Figure 2, the (QBP1-bio)/(SA-QDot) mixed colloid exhibited low affinity toward GaN compared to the silica, as evidenced in the fluorescence images in both geometries and the corresponding differences in the $\mathrm{PL}$ signals (Figure $2 \mathrm{~d}$ ). These results demonstrate specific binding of the peptide-decorated QDots on the silica surface but not on the GaN or gold regions of the chip (see the control experiments in Supporting Information, Figure S1).

In conventional methods, a number of different masking steps are necessary to pattern the QDot film in specific regions on an optoelectronic chip. ${ }^{36-39}$ These procedures, involving additional photolithographic patterning and subsequent removal of the QDot film by etching, add additional masking steps, thereby making the fabrication process laborious. Similarly, the chemical immobilization methods involve many different surface modification steps, ${ }^{40,41}$ again resulting in lengthy procedures. In our approach, we use an actual device, in somewhat complex architecture, containing the three separate material regions of silica, gold, and GaN, as summarized in Figure 3. Following the targeted assembly of the QDots using the silica-binding peptide as the molecular linker, we next demonstrated device performance via the measurement of the PL excitation. It can be claimed that one can create a binary pattern using nonspecific (hydrophobic-hydrophilic) interactions, but for the real chip applications, a more complex selectivity is desired (e.g., in our microchip where we should be able to differentiate gold, silica, and GaN). This can be only achieved by utilizing the specific binding where the specificity implies material selectivity, here brought about by the silica-binding peptide.

The simple LED device is made of InGaN/GaN quantum wells embedded in a vertical $p-i-n$ diode architecture with one metal contact to the $p$-side of the diode, at the top, and another metal contact, to the $\mathrm{n}$-side of the diode, at the bottom. The top view of the fabricated LED shown in the optical image in Figure 3A reveals fairly complex but distinct regions of the metal contacts (yellow), the semiconductor mesa (gray), and the silica film on the top (brownish), where the QDots would be assembled. The hybrid QDot-LED is operated 

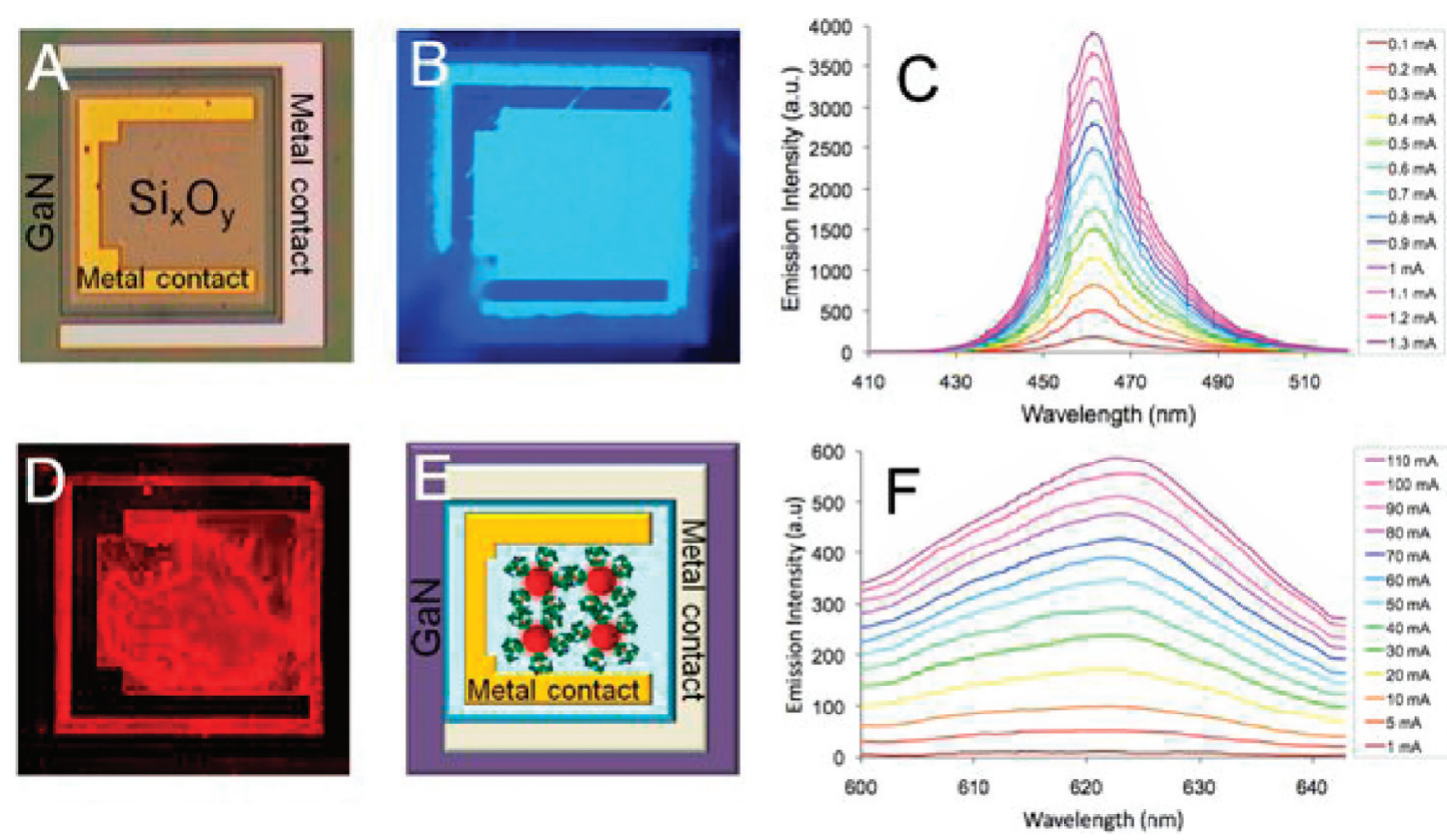

Figure 3. (A) Plan view of the fabricated LED device with microarchitectured metal, semiconductor, and dielectric regions (light optical microscopy). (B) Image and (C) profiles of electroluminescence of the microfabricated LED device at $462 \mathrm{~nm}$ and at various levels of driving current at room temperature. (D) Schematic representation of the LED shown in (A) with the (QBP1bio)/(SA-QDot) hybrid construct targeted assembly only on the silica regions of the device. (E) Fluorescence microscopy image contrasted by light emitting in red at $620 \mathrm{~nm}$, confirming directed self-assembly of the (QBP1-bio)/(SA-QDots) only on the targeted silica surface of the device. (F) Photoluminescence of the hybrid construct targeted assembly on the silica that is optically pumped by the electrically driven LED at room temperature.

by using the LED as the pump light source and assembled QDot layer as the photoluminescent layer. The LED emits in blue when electrically driven at room temperature (Figure 3B) and optically pumps assembled QDots. The performance of the device is demonstrated in Figure 3C, showing the electroluminescence spectra (EL) emitted from the LED at a peak wavelength of $462 \mathrm{~nm}$ in blue. The EL signal is parametrized with respect to the various levels of current injection, ranging from 0.1 to $1.3 \mathrm{~mA}$, at room temperature. Here we observe that, as the current injection is increased, the electroluminescence of the LED is expectedly increased. The purpose of the next step is to self-assemble the QDots selectively on the targeted silica regions of the device. To accomplish this task, we used the procedure of mixing QBP-bio and functionalized the SA-QDot in the colloidal solution, which, as demonstrated above and illustrated in Figures 1 and 2, provides the most successful targeted assembly while distinguishing one material region from another because of the presence of the silicabinding peptides that are material-selective with preference to silica. This is demonstrated in the confocal microscopy image in Figure 3D, which confirms that the red-emitting, peptide-decorated QDots are assembled fairly uniformly on the silica film of the LED, leaving anywhere else black, including the Au and $\mathrm{GaN}$ regions. These results are also schematically illustrated in Figure 3E for clarity, outlining the device architecture; the QDots assemble in the silica regions (light blue), leaving the gold (yellow) and GaN (violet) regions untouched.

In all of the assembly experiments to investigate comparatively the optical quality of the resulting assemblies using these different approaches, we used commercial silica slides with very low surface roughness purchased from the vendor SPI (Pennsylvania). On the other hand, Figure 3D shows the application of the hybrid assembly approach to real LED chip surfaces. During the fabrication of these LEDs, we coated silicon oxide films using plasma-enhanced chemical vapor deposition, which were subsequently patterned using standard photolithography and reactive ion etching. As a result, the film quality of the silica coating on our LED chips is worse than that of the silica slides acquired from SPI. The silica films on our LEDs suffer high surface roughness and irregularities, which in turn give rise to non-uniformity in the assembled QDot film on these LEDs.

It is also possible to optically excite the QDots directly with the electroluminescence of the LED for color conversion by electrically driving it since the redemitting QDots are intimately integrated on the surface of the LED. We carried out such an experiment and obtained the resulting PL spectra from the device, as demonstrated in Figure 3F. In the spectra, the integrated QDots emit in red (at 620-625 nm) upon their optical excitation with the LED electroluminescence (shown Figure 3B). In these PL spectra, the parametrization was carried out at various levels of current 
injection, ranging from 1 to $110 \mathrm{~mA}$, at room temperature. As the current injection to the LED is increased, the PL of these QDots, integrated on the optically pumping LED, is expectedly also increased. These results confirm the successful targeted self-assembly of peptide-decorated QDots on the LED and the subsequent success of optical pumping of the QDots using the EL. Compared to the performance of bare LED emission at $462 \mathrm{~nm}$, the performance of QDot-assembled LED is lower at $620 \mathrm{~nm}$, which is directly related to the photoluminescence coming from the hybridized QDots pumped by the LED. The performance of such QDot-LEDs can be increased by creating layer-by-layer assemblies of QDots, which was achieved in our previous work. ${ }^{16}$

\section{CONCLUSIONS}

In summary, we demonstrated spatially selective self-assembly of the quantum dot nanocrystal emitters functionalized with genetically engineered solid-binding peptides as smart molecular linkers with material- binding specificity on a multimaterial patterned colorconversion LED and demonstrated the successful operation on the device. Here, QBP1 provides a versatile smart molecular linker, selecting one material over others, for the targeted assembly of nanoentities (here QDots). The QBP1-QDot coupling here is accomplished with the strong molecular recognition characteristics of the bio-SA interaction, where biotin is conjugated to QBP1 and strepavidin to QDot, through fairly straightforward processes. Overcoming the limitations imposed by masking, lithography, chemical functionalization, and providing material specificity, the solid-binding peptides, when used as molecular linkers as demonstrated here for LED devices, could potentially open up new opportunities for controlled assembly of functional nanoentities (such as quantum dots, nanoparticles, nanowires, nanotubes, etc.) targeted and directly assembled on multimaterial microand nanopatterned surfaces toward building novel biomolecular-based functional hybrid electronic, photonic, and magnetic devices.

\section{METHODS}

Peptides and Buffer Solutions. Biotinylated QBP1 peptide obtained from a knowledge-based method was synthesized using standard Fmoc solid-phase peptide synthesis techniques and purified using C-18 reverse-phase liquid chromatography (RPLC) to a level of $>95 \%$ purity. The peptide was synthesized without a blocking group either at the $-\mathrm{N}$ or $-\mathrm{C}$ termini. The peptide solution was prepared in $\mathrm{PC}$ buffer $\left(55 \mathrm{mM} \mathrm{KH}_{2} \mathrm{PO}_{4}, 45\right.$ $\mathrm{mM} \mathrm{Na}{ }_{2} \mathrm{CO}_{3}$, and $200 \mathrm{mM} \mathrm{NaCl}$ ).

Preparation of Substrates and Specificity Experiments. We prepared $\mathrm{Au} /$ silica and GaN/silica samples for cross-specificity experiments. For the Au/silica experiments, we prepared two different samples, one with silica patterns on the gold layer and the other with the gold patterns on silica layer. For the GaN/silica sample, we deposited $300 \mathrm{~nm}$ thick silica on the GaN surface and then patterned it using a mask aligner followed by etching. The details of preparation of the substrates are given in Supporting Information. After the sample preparation, we drop-casted (QBP1-bio)/(SA-QDot) hybridized nanoassemblies on the Au/ silica and $\mathrm{GaN} /$ silica surfaces and washed each of the samples with the procedure described above. Next, we measured the photoluminescence with a monochromator with $2000 \mu \mathrm{m}$ slit width and $500 \mathrm{~ms}$ integration time by using $325 \mathrm{~nm} \mathrm{HeCd}$ laser. For the uniformity of the nanocrystal film, we recorded data from three different locations of the same sample. Moreover, we repeated the experiment with the samples prepared in the same conditions at least three times for reproducibility.

Assembly of Quantum Dots on Color-Conversion Light-Emitting Diodes. The streptavidin-coated Evifluors quantum dot conjugates were acquired from Evident Technologies (Troy, NY). A maple redorange quantum dot, with an emission peak at $620 \mathrm{~nm}$, was used. Then the QDots were activated with streptavidin molecules to couple with biotin. For the sample preparation, equal volumes $(15-30 \mu \mathrm{L})$ of $100 \mu \mathrm{g} / \mathrm{mL}$ peptide solutions and $2.5 \mu \mathrm{M}$ SA-QDots were used for each quartz chip. The streptavidincoated nanocrystals were coated with biotinylated QBP1; this was achieved by optimizing the concentration and volume ratio of QBP1-bio and SA-QDots. Decorating the outer surface of SAQDots with QBP1-bio makes SA-QDots easily bind to the silica surface. Peptide-conjugated QDots were assembled on LED surfaces and incubated. The details of LED preparation and assembly of QDot on LED surface can be found in Supporting Information.

Acknowledgment. This work was supported, in part, by EUN4E NoE, ESF EURYI, and TUBITAK (106E020, 107E088, 109E002, 109E004, 110E010) at Bilkent; by TUBITAK and by NSF-MRSEC (GEMSEC) and NSF BioMat programs at UW (C.T. and M.S.). GEMSEC-SECF is part of an MRSEC-MRFN, Materials Research Facilities Network. H.V.D. further acknowledges additional support from the Turkish National Academy of Sciences Distinguished Young Scientist Award and Singapore NRF Fellowship.

Supporting Information Available: Details of LED fabrication, silica/GaN gold/silica substrate preparation, details of surface plasmon resonance experiment. This material is available free of charge via the Internet at http://pubs.acs.org.

\section{REFERENCES AND NOTES}

1. Morello, A.; Pla, J. J.; Zwanenburg, F. A.; Chan, K. W.; Tan, K. Y.; Huebl, H.; Mottonen, M.; Nugroho, C. D.; Yang, C. Y.; van Donkelaar, J. A.; et al. Single-Shot Readout of an Electron Spin in Silicon. Nature 2010, 467, 687-691.

2. Klimov, V. I.; Ivanov, S. A.; Nanda, J.; Achermann, M.; Bezel, I.; McGuire, J. A.; Piryatinski, A. Single-Exciton Optical Gain in Semiconductor Nanocrystals. Nature 2007, 447, 441446.

3. Gur, I.; Fromer, N. A.; Geier, M. L.; Alivisatos, A. P. Air-Stable All-Inorganic Nanocrystal Solar Cells Processed from Solution. Science 2005, 310, 462-465.

4. Norris, D. J.; Sacra, A.; Murray, C. B.; Bawendi, M. G. Measurement of the Size-Dependent Hole Spectrum in CdSe Quantum Dots. Phys. Rev. Lett. 1994, 72, 2612-2615.

5. Jin, Y. D.; Gao, X. H. Plasmonic Fluorescent Quantum Dots. Nat. Nanotechnol. 2009, 4, 571-576.

6. Schubert, E. F.; Hunt, N. E.; Micovic, M.; Malik, R. J.; Sivco, D. L.; Cho, A. Y.; Zydzik, G. J. Highly Efficient Light-Emitting Diodes with Microcavities. Science 1994, 265, 943-5.

7. Nizamoglu, S.; Ozel, T.; Sari, E.; Demir, H. V. White Light Generation Using CdSe/ZnS Core-Shell Nanocrystals Hybridized with InGaN/GaN Light Emitting Diodes. Nanotechnology 2007, 18, 065709. 
8. Erdem, T.; Nizamoglu, S.; Sun, X. W.; Demir, H. V. A Photometric Investigation of Ultra-efficient LEDs with High Color Rendering Index and High Luminous Efficacy Employing Nanocrystal Quantum Dot Luminophores. Opt. Express 2010, 18, 340-347.

9. Jang, E.; Jun, S.; Jang, H.; Llim, J.; Kim, B.; Kim, Y. WhiteLight-Emitting Diodes with Quantum Dot Color Converters for Display Backlights. Adv. Mater. 2010, 22, 30763080.

10. Ali, M.; Chattopadhyay, S.; Nag, A.; Kumar, A.; Sapra, S.; Chakraborty, S.; Sarma, D. D. White-Light Emission from a Blend of CdSeS Nanocrystals of Different Se:S Ratio. Nanotechnology 2007, 18, 075401.

11. Lee, B.; Kim, Y.; Lee, S.; Kim, Y. S.; Wang, D. Y.; Cho, J. Layerby-Layer Growth of Polymer/Quantum Dot Composite Multilayers by Nucleophilic Substitution in Organic Media. Angew. Chem., Int. Ed. 2010, 49, 359-363.

12. Li, S.; Toprak, M. S.; Jo, Y. S.; Dobson, J.; Kim, D. K.; Muhammed, M. Bulk Synthesis of Transparent and Homogeneous Polymeric Hybrid Materials with ZnO Quantum Dots and PMMA. Adv. Mater. 2007, 19, 4347-4352.

13. Gupta, S.; Uhlmann, P.; Agrawal, M.; Lesnyak, V.; Gaponik, N.; Simon, F.; Stamm, M.; Eychmuller, A. Covalent Immobilization of Quantum Dots on Macroscopic Surfaces Using Poly(acrylic acid) Brushes. J. Mater. Chem. 2008, 18, 214-220.

14. Cicek, N.; Nizamoglu, S.; Ozel, T.; Mutlugun, E.; Karatay, D. U.; Lesnyak, V.; Otto, T.; Gaponik, N.; Eychmuller, A.; Demir, H. V. Structural Tuning of Color Chromaticity through Nonradiative Energy Transfer by Interspacing CdTe Nanocrystal Monolayers. Appl. Phys. Lett. 2009, 94, 061105.

15. Komarala, V. K.; Rakovich, Y. P.; Bradley, A. L.; Byrne, S. J.; Corr, S. A.; Gunko, Y. K. Emission Properties of Colloidal Quantum Dots on Polyelectrolyte Multilayers. Nanotechnology 2006, 17, 4117-4122.

16. Nizamoglu, S.; Demir, H. V. Hybrid White Light Sources Based on Layer-by-Layer Assembly of Nanocrystals on Near-UV Emitting Diodes. Nanotechnology 2007, 18, 1-4.

17. Xia, Y. N.; Whitesides, G. M. Soft Lithography. Annu. Rev. Mater. Sci. 1998, 28, 153-184.

18. Michel, B.; Bernard, A.; Bietsch, A.; Delamarche, E.; Geissler, M.; Juncker, D.; Kind, H.; Renault, J. P.; Rothuizen, H.; Schmid, H.; et al. Printing Meets Lithography: Soft Approaches to High-Resolution Printing. IBM J. Res. Dev. 2001, 45, 697-719.

19. Tang, L.; Kocabas, S. E.; Latif, S.; Okyay, A. K.; Ly-Gagnon, D. S.; Saraswat, K. C.; Miller, D. A. B. Nanometre-Scale Germanium Photodetector Enhanced by a Near-Infrared Dipole Antenna. Nat. Photonics 2008, 2, 226-229.

20. Lee, B. G.; Biberman, A.; Chan, J.; Bergman, K. HighPerformance Modulators and Switches for Silicon Photonic Networks-on-Chip. IEEE J. Sel. Top. Quantum Electron. 2010, 16, 6-22.

21. Lee, C. J.; Lin, J. F. Lens Designs by Ray Tracing Analyses for High-Performance Reflection Optical Modules. J. Opt. 2010, 12, 1-9.

22. Liu, J. S.; Tanaka, T.; Sivula, K.; Alivisatos, A. P.; Frechet, J. M. J. Employing End-Functional Polythiophene To Control the Morphology of Nanocrystal-Polymer Composites in Hybrid Solar Cells. J. Am. Chem. Soc. 2004, 126, 65506551.

23. Ohira, K.; Kobayashi, K.; lizuka, N.; Yoshida, H.; Ezaki, M.; Uemura, H.; Kojima, A.; Nakamura, K.; Furuyama, H.; Shibata, H. On-Chip Optical Interconnection by Using Integrated III-V Laser Diode and Photodetector with Silicon Waveguide. Opt. Express 2010, 18, 15440-15447.

24. Fischbein, M. D.; Drndic, M. CdSe Nanocrystal Quantum-Dot Memory. Appl. Phys. Lett. 2005, 86, 193106-1-193106-3.

25. Sarikaya, M.; Tamerler, C.; Jen, A. K. Y.; Schulten, K.; Baneyx, F. Molecular Biomimetics: Nanotechnology through Biology. Nat. Mater. 2003, 2, 577-585.

26. Whaley, S. R.; English, D. S.; Hu, E. L.; Barbara, P. F.; Belcher, A. M. Selection of Peptides with Semiconductor Binding
Specificity for Directed Nanocrystal Assembly. Nature 2000, 405, 665-668.

27. Sano, K.; Shiba, K. A Hexapeptide Motif That Electrostatically Binds to the Surface of Titanium. J. Am. Chem. Soc. 2003, 125, 14234-14235.

28. Slocik, J. M.; Naik, R. R. Probing Peptide-Nanomaterial Interactions. Chem. Soc. Rev. 2010, 39, 3454-3463.

29. Slocik, J. M.; Stone, M. O.; Naik, R. R. Synthesis of Gold Nanoparticles Using Multifunctional Peptides. Small 2005, 1, 1048-1052.

30. Sano, K.; Sasaki, H.; Shiba, K. Utilization of the Pleiotropy of a Peptidic Aptamer To Fabricate Heterogeneous Nanodot-Containing Multilayer Nanostructures. J. Am. Chem. Soc. 2006, 128, 1717-1722.

31. Huang, Y.; Chiang, C. Y.; Lee, S. K.; Gao, Y.; Hu, E. L.; De Yoreo, J.; Belcher, A. M. Programmable Assembly of Nanoarchitectures Using Genetically Engineered Viruses. Nano Lett. 2005, 5, 1429-1434.

32. Bhaviripudi, S.; Qi, J.; Hu, E. L.; Belcher, A. M. Synthesis, Characterization, and Optical Properties of Ordered Arrays of III-Nitride Nanocrystals. Nano Lett. 2007, 7, 35123517.

33. Oren, E. E.; Tamerler, C.; Sahin, D.; Hnilova, M.; Seker, U. O. S.; Sarikaya, M.; Samudrala, R. A Novel Knowledge Based Approach To Design Inorganic-Binding Peptides. Bioinformatics 2007, 23, 2816-2822.

34. Xiong, R. L.; Li, Z.; Mi, L.; Wang, P. N.; Chen, J. Y.; Wang, L. X.; Yang, W. L. Study on the Intracellular Fate of Tat PeptideConjugated Quantum Dots by Spectroscopic Investigation. J. Fluoresc. 2010, 20, 551-556.

35. Liu, C.; Chung, S. Y.; Lee, S.; Weiss, S.; Neuhauser, D. Adsorbate-Induced Absorption Redshift in an Organic-Inorganic Cluster Conjugate: Electronic Effects of Surfactants and Organic Adsorbates on the Lowest Excited States of a Methanethiol-CdSe Conjugate. J. Chem. Phys. 2009, 131, 174705.

36. Guo, W.; Guico, R. S.; Beresford, R.; Xu, J. M. Growth of Highly Ordered Relaxed InAs/GaAs Quantum Dots on Non-Litho Graphically Patterned Substrates by Molecular Beam Epitaxy. J. Cryst. Growth 2006, 287, 509-513.

37. Larsson, M.; Wallin, D.; Xu, H. Q. A Highly Tunable Lateral Quantum Dot Realized in InGaAs/InP by an Etching Technique. J. Appl. Phys. 2008, 103, 086101.

38. Grutzmacher, D.; Fromherz, T.; Dais, C.; Stangl, J.; Muller, E.; Ekinci, Y.; Solak, H. H.; Sigg, H.; Lechner, R. T.; Wintersberger, E.; et al. Three-Dimensional Si/Ge Quantum Dot Crystals. Nano Lett. 2007, 7, 3150-3156.

39. Kalliakos, S.; Garcia, C. P.; Pellegrini, V.; Zamfirescu, M.; Cavigli, L.; Gurioli, M.; Vinattieri, A.; Pinczuk, A.; Dennis, B. S.; Pfeiffer, L. N.; et al. Photoluminescence of Individual Doped GaAs/AIGaAs Nanofabricated Quantum Dots. Appl. Phys. Lett. 2007, 90, 181902.

40. Qian, J.; Zhang, C. Y.; Cao, X. D.; Liu, S. Q. Versatile Immunosensor Using a Quantum Dot Coated Silica Nanosphere as a Label for Signal Amplification. Anal. Chem. 2010, 82, 6422-6429.

41. Constantine, C. A.; Gattas-Asfura, K. M.; Mello, S. V.; Crespo, G.; Rastogi, V.; Cheng, T. C.; DeFrank, J. J.; Leblanc, R. M. Layer-byLayer Biosensor Assembly Incorporating Functionalized Quantum Dots. Langmuir 2003, 19, 9863-9867. 